\title{
Aortocoronary dissection complicating angioplasty of chronically occluded right coronary arteries: Is a conservative approach the right approach?
}

\author{
Ian Hunt, MRCS, ${ }^{\text {a }}$ Matthew E. Faircloth, MRCP, ${ }^{\text {b }}$ Pranava Sinha, MCh, ${ }^{\text {a }}$ Michael S. Marber, PhD, FRCP, ${ }^{\text {b }}$ \\ Graham E. Venn, MS, FRCS, ${ }^{a}$ and Christopher P. Young, MS, FRCS, ${ }^{a}$ London, United Kingdom
}

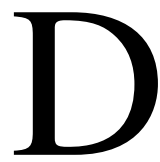
issection of the proximal aorta during coronary angiography is a well-recognized and rare complication that occurs more frequently during canalization of a chronically occluded right coronary artery (RCA). Most reported cases describe conservative management or use of intracoronary stents if the entry point is within the coronary artery. Early surgical intervention is rarely advocated or described and might reflect a reporting bias in the cardiology literature. Any patient in whom there is a significant ascending aortic flap should undergo immediate surgical intervention.

\section{Clinical Summary}

PATIENT 1. A 62-year-old woman underwent percutaneous coronary intervention (PCI) for chronic stenoses of the RCA. She had a 1-year history of angina and preserved ventricular function. The RCA was cannulated without difficulty, and the lesion was crossed and dilated. Several attempts were made to pass intracoronary stents, and eventually $3.5-\mathrm{mm}$ stents were placed across proximal and distal lesions with a good angiographic result. At this stage, a dissection flap at the ostium of the RCA was noted, and contrast was clearly seen throughout the wall of the ascending aorta (Figure 1, arrows). A further 3.5-mm stent was deployed at the ostium with apparent success. Echocardiography demonstrated a false lumen in the ascending aorta but no pericardial effusion.

After transfer to the coronary care unit, the patient was treated conservatively but the next day experienced intrascapular back pain and inferior electrocardiographic changes. A chest computed tomogram demonstrated an aortic dissection from the ascending aorta to the distal descending aorta. The patient underwent emergency replacement of the ascending aorta and coronary artery bypass grafting to the mid-RCA and conus branch. At the time of the operation, the dissection originated at the RCA ostium and involved the whole of the ascending, arch, and descending aorta, as well as the noncoronary and right coronary cusps. The aortic valve was resuspended. The patient was returned to the intensive care unit on inotropes.

From the Departments of Cardiac Surgery ${ }^{\mathrm{a}}$ and Cardiology, ${ }^{\mathrm{b}}$ St Thomas' Hospital, London, United Kingdom.

Received for publication Aug 5, 2005; accepted for publication Aug 17, 2005.

Address for reprints: Ian Hunt, MRCS, Cardiothoracic Surgery Centre, St Thomas' Hospital, London, SE1 7EH, UK (E-mail: ian.hunt@gstt.nhs.uk).

J Thorac Cardiovasc Surg 2006;131:230-1

$0022-5223 / \$ 32.00$

Copyright $\odot 2006$ by The American Association for Thoracic Surgery

doi:10.1016/j.jtcvs.2005.08.051

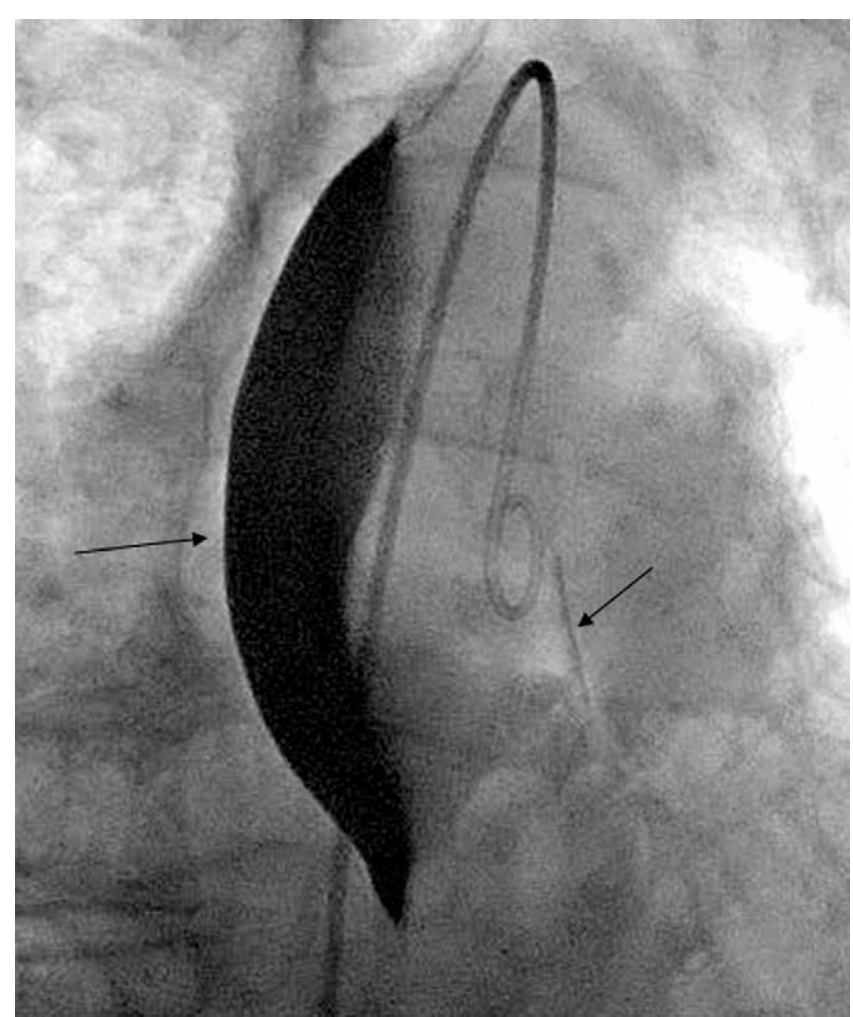

Figure 1. Proximal aortic dissection, with contrast clearly seen filling the false lumen on both sides of the aortic wall (arrows).

PATIENT 2. A 73-year-old man with progressive exertional angina underwent elective PCI to a chronically occluded RCA. However, a guide wire could not be passed across the occluded vessel, and the RCA ostium was dissected (Figure 2; white arrow = coronary dissection, black arrow $=$ retrograde aortic dissection clearly seen). Despite multiple attempts to re-enter the true lumen, the procedure was abandoned. The patient remained pain free and stable throughout the procedure. Echocardiography demonstrated no evidence of pericardial fluid or tamponade.

He was again transferred to the coronary care unit for conservative management. Overnight, the patient complained of intrascapular discomfort and become progressively hypertensive and was started on labetalol infusion. Once again, the following day a chest computed tomogram was ordered and demonstrated aortic dissection starting at the root of the aorta and extending into the brachiocephalic artery through the arch of the aorta into the 


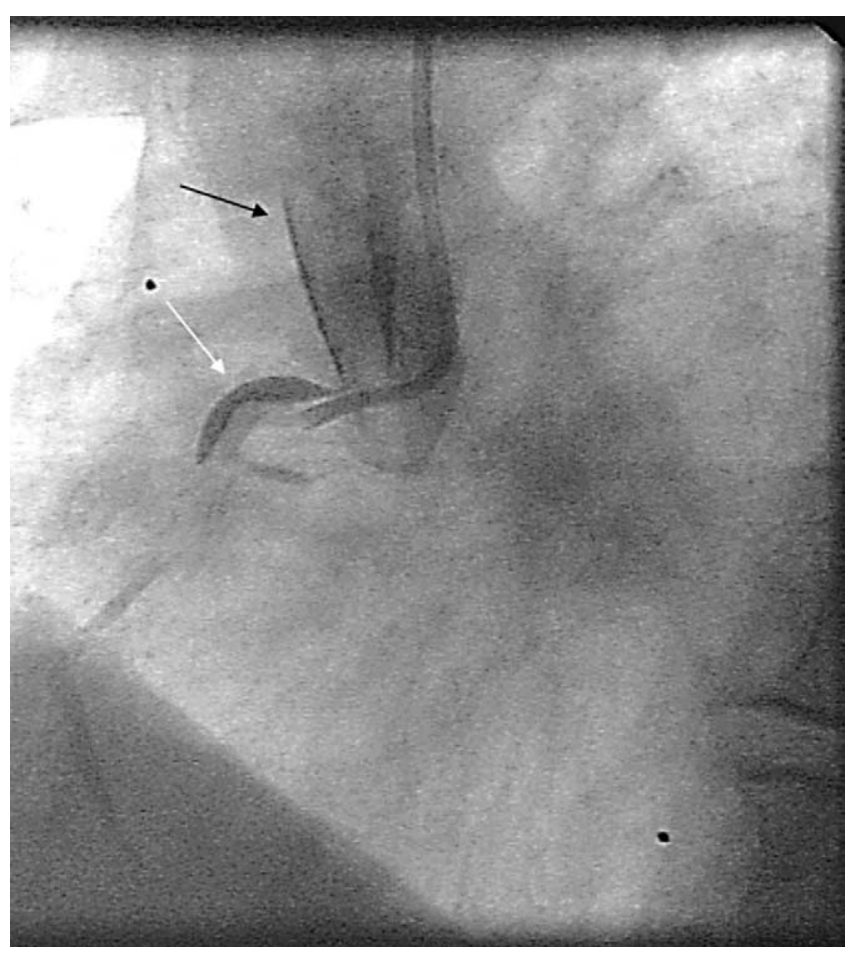

Figure 2. Coronary dissection at the RCA ostium (white arrow), with retrograde contrast seen in the aortic wall (black arrow).

descending aorta, stopping just before the renal arteries. The patient underwent emergency replacement of the ascending aorta and resuspension of the aortic valve. Inspection revealed the dissection to be originating from within the RCA. He was returned to the intensive care unit. Both patients remain well at 3 months' follow-up.

\section{Discussion}

Iatrogenic aortic dissection during coronary angiography is a rare but recognized occurrence. ${ }^{1,2}$ It is typically described in the context of attempted recanalization of a chronically occluded RCA. ${ }^{3}$ The likely cause after PCI is retrograde extension to the sinus of Valsalva and ascending aorta after localized coronary dissection, which might also extend antegradely. ${ }^{4}$ Isolated case reports describe conservative management of limited aortocoronary dissection or use of intracoronary stenting at the entry point. ${ }^{1,3-5}$ Surgical intervention is described rarely and only in the context of an extending ascending aortic dissection. ${ }^{2}$ The previous reports identified in the cardiology literature (and published exclusively in cardiology journals) is likely to reflect a reporting bias and might have led to adoption of a more conservative approach to such a complication than one would expect. If the dissection is limited to the immediate area of the RCA origin, then conservative management might be appropriate, providing a surgical ream is informed and involved from the start. The decision should be reversed if the patient experienced symptoms or signs of a further dissection.

\section{References}

1. Bae JH, Kim KB, Kim KS, Kim YN. A case of aortocoronary dissection as a complication during a percutaneous transluminal coronary angioplasty (PTCA). Int J Cardiol. 1998;66:237-40.

2. Dunning DW, Kahn JK, Hawkins ET, O'Neill WW. Iatrogenic coronary artery dissections extending into and involving the aortic root. Catheter Cardiovasc Interv. 2000;51:387-93.

3. Pentousis D, Toussaint M, Zheng H, Favereau X, Guerin Y, Bardet A, et al. Conservative management for an extensive type A aortic dissection complicating coronary angioplasty. J Invasive Cardiol. 2000;12: 320-3.

4. Kostov J, Stankovic G. Aortic root and extensive coronary dissections complicating recanalization of chronic right coronary artery occlusion: refraining from stenting may have a favorable outcome! J Invasive Cardiol. 2003;15:164-6.

5. Sutton AG, Aggarwal RK, de Belder MA. Type A dissection of the ascending thoracic aorta during percutaneous coronary intervention. J Invasive Cardiol. 2000;12:147-50. 